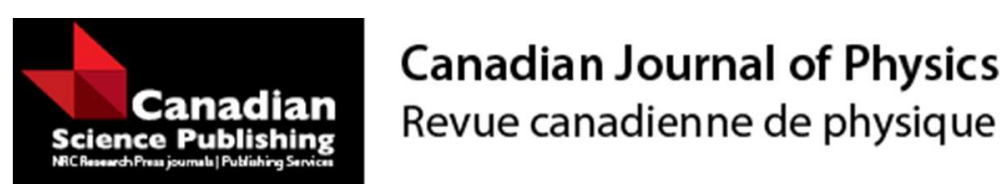

\title{
Higgs particles interacting via a scalar Dark Matter field
}

\begin{tabular}{|r|l|}
\hline Journal: & Canadian Journal of Physics \\
\hline Manuscript ID & cjp-2016-0467.R1 \\
\hline Manuscript Type: & Article \\
\hline Date Submitted by the Author: & 15 -Oct-2016 \\
\hline Complete List of Authors: & $\begin{array}{l}\text { Bhattacharya, Yajnavalkya; York University, Physics and Astronomy; New } \\
\text { Jersey Institute of Technology, The Center for Solar-Terrestrial Research } \\
\text { Darewych, Jurij; Department of Physics and Astronomy, }\end{array}$ \\
\hline Keyword: & Dark Matter, Higgs, bound state, variational, Quantum Field Theory \\
\hline
\end{tabular}

\section{SCHOLARONE ${ }^{\text {m }}$}

Manuscripts 


\title{
Higgs particles interacting via a scalar Dark Matter field
}

\author{
Yajnavalkya Bhattacharya \\ Jurij W. Darewych \\ York University, Toronto, Canada \\ darewych@yorku.ca
}

November 2, 2016

\begin{abstract}
We study a system of two Higgs bound state, interacting via a real scalar Dark Matter mediating field, without imposing $Z_{2}$ symmetry on the DM sector of the postulated Lagrangian. The variational method in the Hamiltonian formalism of QFT is used to derive relativistic wave equations for the two-Higgs system, using a truncated Fock-space trial state. Approximate solutions of the 2-body relativistic coupled integral equations are presented, and conditions for the existence of Higgs bound states is examined in a broad parameter space of DM mass and coupling constants.
\end{abstract}

Keywords: Scalar Dark Matter, Higgs, bound state, Quantum Field Theory, variational method.

\section{Introduction}

Astrophysical observations of gravitational effects at all length scales of the universe, including our Milky Way galaxy, indicate the existence of Dark Matter (DM) [1, 2, 3]. To date, DM has been observed only through its gravitational influence such as rotation curves of galaxies, mismatch of estimated mass and luminous matter in galaxy clusters, lensing of galactic 
Supernovae of Type Ia and Cosmic Microwave Background images, etc. A major open question is the nature of DM, since to date, there are no direct measurements of DM's electroweak, or any non-gravitational, interactions.

One conjecture is that DM is made up non-baryonic particles called WIMPs (Weakly Interacting Massive Particles). Experimental efforts at detecting DM, including WIMPs, are ongoing [4]. The literature on this subject is extensive. A brief, useful overview of DM and efforts to detect them is given in ref. [5].

An outstanding question is how DM interacts with Standard Model particles, including the Higgs particles. There have been a number of theoretical models that address this problem (see, for example, references $[6,7,8]$ and citations therein). Recently, Petersson et al. have proposed a Supersymmetric generalization of the Standard Model in which the Higgs boson can disintegrate into a photon and DM particles [9].

The possibility of Higgs bound states ("Higgsonium") has been examined well before the experimental detection of the Higgs boson (cf. references [10] to [14] ). In these prior studies, the domain of the Higgs mass and coupling strength for which Higgsonium binding might occur, were investigated. These 2-Higgs bound-state results (due to Higgs self coupling) are somewhat moot, now that the Standard Model Higgs mass has been observed to be 125.7 $\mathrm{GeV}$.

The present work is concerned with the interactions of Higgs particles with DM particles. In particular we shall examine the possibility of twobody Higgs bound states due a mediating DM field. To our knowledge, this is the first investigation of Higgs relativistic bound state formation, using a general DM Lagrangian, via a singlet scalar DM channel.

\section{The model}

In this work the Dark Matter is assumed to be a spinless, singlet, massive scalar field $\phi$, of mass $\mu$, coupled with the Higgs field $\chi$, which is taken to be the Higgs sector of the Standard Model. Models with scalar singlet DM have been studied previously (see for e.g. references [16] [17]). In this study, we shall consider the interaction of the Higgs particles with DM only. The interaction terms will be taken to be of a general form consistent with classical stability and renormalizability, that is the postulated Lagrangian density of this model is $(\hbar=c=1)$ 


$$
\begin{array}{r}
\mathcal{L}=\frac{1}{2} \partial^{\nu} \phi \partial_{\nu} \phi-\frac{1}{2} \mu^{2} \phi^{2}-\sigma \phi^{3}-\kappa \phi^{4} \\
+\frac{1}{2} \partial^{\nu} \chi \partial_{\nu} \chi-\frac{1}{2} m^{2} \chi^{2}-\lambda v \chi^{3}-\frac{1}{4} \lambda \chi^{4} \\
-g_{1} \chi \phi^{2}-\eta_{1} \chi^{2} \phi^{2}-\eta_{2} \chi \phi^{3}-\eta_{3} \chi^{3} \phi-g_{2} \chi^{2} \phi
\end{array}
$$

where $\kappa, \lambda, g_{1}, g_{2}, v$ and $\eta_{j}(j=1,2,3)$ are positive coupling constants; $\lambda, \kappa, \eta_{j}$ being dimensionless, and $v, \sigma, g_{j}$, having dimensions of mass. Aside from the Higgs sector coupling constants $\lambda$ and $v$, all the others are presently unknown and will be treated to be adjustable.

Note that we have not included a $g \chi \phi$ term in our postulated Lagrangian (1) becase such a mixing term would contain a coupling constant $g$ with dimensions of (mass) ${ }^{2}$, which is unusual and would allow for instability of the DM.

The proposed Lagrangian (1) does allows for the decay of a very heavy single DM particle into two Higgs, provided that $m_{D M} \geq 2 m_{\text {Higgs }}$, where $m_{D M} \equiv \mu$, and $m_{\text {Higgs }} \equiv m$ are the DM and Higgs masses respectively.

In order to decrease the coupling-constant parameter space we shall set $\sigma=\kappa=\eta_{i}=0$ for this paper.

It is shown below that in the domain where the effective Higgs-DM dimensionless coupling constant varies from 0-2, two Higgs bound states are formed only if $m_{D M} \leq m_{\text {Higgs }}$, which excludes DM decay into the Higgs.

Models in which the DM is represented by a scalar with an assumed $Z_{2}$ symmetry, have been investigated by many authors, e.g. [16, 18, 19, 21]. Our Lagrangian, however, does not impose a $Z_{2}$ symmetry, since the DM particle is represented by a spinless real scalar - thus allowing for trilinear terms, such as $g_{2} \chi^{2} \phi$, in which the dimensionful coupling constant is similar to that of the Higgs sector of the DM.

Our choice is motivated by the fact that the popularity of the $Z_{2}$ symmetry has largely resulted in the avoidance of studying the simplest case of a real scalar DM with no additional internal degrees of freedom. In an article on scalar DM, Rodejohann and Yaguna [22] have noted that "..use of discrete symmetries is questionable not only due to its lack of motivation, but also because they are expected to be broken by gravitational effects at the Planck scale, including dark matter decay and likely destroying the feasibility of such models. That is why it is often implicitly assumed that such 
a discrete symmetries are actually the remnants of additional gauge or flavor symmetries present at a higher scale, thereby delegating the problem to a framework larger than the model under consideration." In another recent paper, Cirelli [23] notes that "The "stabilization symmetry" has become such a household tool for the model builder that often he/she does not even spend time arguing about it: when in a hurry, just say that you add a $Z_{2}$ symmetry and move on."

\section{Quantization and Hamiltonian Formalism}

Upon canonical quantization (in the interaction picture) the classical fields $\phi, \chi$ become operators

$$
\begin{array}{r}
\phi(x)=\int \frac{d^{3} p}{\sqrt{(2 \pi)^{3} 2 \omega(p, \mu)}}\left[d(\mathbf{p}) e^{-i p_{\mu} \cdot x}+d^{\dagger}(\mathbf{p}) e^{i p_{\mu} \cdot x}\right], \\
\chi(x)=\int \frac{d^{3} p}{\sqrt{(2 \pi)^{3} 2 \omega(p, m)}}\left[h(\mathbf{p}) e^{-i p_{m} \cdot x}+h^{\dagger}(\mathbf{p}) e^{i p_{m} \cdot x}\right],
\end{array}
$$

where $\quad \omega(p, m)=\sqrt{\mathbf{p}^{2}+m^{2}}, \quad \omega(p, \mu)=\sqrt{\mathbf{p}^{2}+\mu^{2}}, \quad x=(t, \mathbf{r}), \quad p_{m}=$ $[\omega(p, m), \mathbf{p}]$ and $p_{\mu}=[\omega(p, \mu), \mathbf{p}]$. The DM and Higgs operators $d^{\dagger}, d$ and $h^{\dagger}, h$ satisfy the usual commutation rules,

$$
\left[d(\mathbf{p}), d^{\dagger}(\mathbf{q})\right]=\delta^{3}(\mathbf{p}-\mathbf{q}) \text { and }\left[h(\mathbf{p}), h^{\dagger}(\mathbf{q})\right]=\delta^{3}(\mathbf{p}-\mathbf{q}),
$$

and all others vanish.

In the Hamiltonian formalism of QFT, the equations to be solved are

$$
\hat{P}^{\beta}|\Psi\rangle=Q^{\beta}|\Psi\rangle
$$

where $\hat{P}^{\beta}=(\hat{H}, \hat{\mathbf{P}})$ and $Q^{\beta}=(E, \mathbf{Q})$ are the energy-momentum operator and corresponding eigenvalues. The $\beta=0$ (energy) component of equation (5) is generally impossible to solve and this applies to the present model. Thus, approximate solutions need to be obtained. We shall use the variational method, which is applicable to strongly coupled systems. This method is based on the principle

$$
\left\langle\delta \Psi_{\text {trial }}|\hat{H}-E| \Psi_{\text {trial }}\right\rangle_{t=0}=0,
$$

where $\hat{H}$ is normal ordered, and $\left|\Psi_{\text {trial }}\right\rangle$ is a suitable trial state. The subscript $t=0$ in equation (6) indicates transformation to the Schrödinger picture, which is convenient for bound states. 


\section{Trial state and channel equations}

For a system of two Higgs particles interacting via the DM field, the simplest trial state that yields tractable, non-trivial results is

$$
\begin{gathered}
\left|\psi_{\text {trial }}\right\rangle=\int d \mathbf{p}_{\mathbf{1}} d \mathbf{p}_{\mathbf{2}} F_{1}\left(\mathbf{p}_{\mathbf{1}}, \mathbf{p}_{\mathbf{2}}\right) h^{\dagger}\left(\mathbf{p}_{\mathbf{1}}\right) h^{\dagger}\left(\mathbf{p}_{\mathbf{2}}\right)|0\rangle+ \\
\int d \mathbf{p}_{\mathbf{1}} d \mathbf{p}_{\mathbf{2}} d \mathbf{p}_{\mathbf{3}} F_{2}\left(\mathbf{p}_{\mathbf{1}}, \mathbf{p}_{\mathbf{2}}, \mathbf{p}_{\mathbf{3}}\right) h^{\dagger}\left(\mathbf{p}_{\mathbf{1}}\right) h^{\dagger}\left(\mathbf{p}_{\mathbf{2}}\right) d^{\dagger}\left(\mathbf{p}_{\mathbf{3}}\right)|0\rangle,
\end{gathered}
$$

where $h$ denotes Higgs and $d$ Dark Matter, and $F_{i},(i=1,2)$ are variational channel wave functions to be determined. We shall work in the rest frame in which $\hat{\mathbf{P}}\left|\psi_{\text {trial }}\right\rangle=0$, which implies that $\mathbf{p}_{\mathbf{1}}+\mathbf{p}_{\mathbf{2}}=0$ in $F_{1}\left(\mathbf{p}_{1}, \mathbf{p}_{2}\right)$ and $\mathbf{p}_{1}+\mathbf{p}_{2}+\mathbf{p}_{3}=0$ in $\left.F_{1}\left(\mathbf{p}_{1}, \mathbf{p}_{2}, \mathbf{p}_{3}\right)\right)$. Substituting the expression (7) into equation (6), evaluating the indicated matrix elements and performing the variations, leads to the following equation for the channel trial functions $F_{1}$ and $F_{2}$ :

$F_{1}\left(\mathbf{q}_{\mathbf{1}},-\mathbf{q}_{\mathbf{1}}\right)\left[2 \omega\left(\mathbf{q}_{\mathbf{1}}, m\right)-E\right]=-4 g_{2} \int \mathrm{d} \mathbf{p} \frac{F_{2}\left(-\mathbf{q}_{\mathbf{1}}, \mathbf{q}_{\mathbf{1}}+\mathbf{p}, \mathbf{p}\right)}{\left(\sqrt{8 \pi^{3}}\right)^{3} \sqrt{2 \omega\left(\mathbf{q}_{\mathbf{1}}, m\right)} \sqrt{2 \omega(\mathbf{p}, \mu)} \sqrt{2 \omega\left(\mathbf{q}_{\mathbf{1}}+\mathbf{p}, m\right)}}$

and

$$
\begin{gathered}
F_{2}\left(\mathbf{q}_{\mathbf{1}}, \mathbf{q}_{\mathbf{2}}, \mathbf{q}_{\mathbf{1}}+\mathbf{q}_{\mathbf{2}}\right)\left[\omega\left(\mathbf{q}_{\mathbf{1}}, m\right)+\omega\left(\mathbf{q}_{\mathbf{2}}, m\right)+\omega\left(\mathbf{q}_{\mathbf{1}}+\mathbf{q}_{\mathbf{2}}, \mu\right)-E\right] \\
=-4 g_{2} \frac{F_{1}\left(-\mathbf{q}_{\mathbf{2}}, \mathbf{q}_{\mathbf{2}}\right)}{\left(\sqrt{\left(8 \pi^{3}\right.}\right)^{3} \sqrt{2 \omega\left(\mathbf{q}_{\mathbf{1}}, m\right)} \sqrt{2 \omega\left(\mathbf{q}_{\mathbf{1}}+\mathbf{q}_{\mathbf{2}}, \mu\right)} \sqrt{2 \omega\left(-\mathbf{q}_{\mathbf{2}}, m\right)}}
\end{gathered}
$$

It is not possible to obtain exact analytic solutions of the coupled, relativistic equations (8) and (9), so we shall resort to obtaining approximate variational-perturbative solutions.

In lowest order approximation we take $E \simeq \omega\left(\mathbf{q}_{\mathbf{1}}, m\right)+\omega\left(\mathbf{q}_{\mathbf{2}}, m\right)$ in equation (9), whereupon equation (9) simplifies to

$$
\begin{array}{r}
F_{2}\left(\mathbf{q}_{\mathbf{1}}, \mathbf{q}_{\mathbf{2}}, \mathbf{q}_{\mathbf{1}}+\mathbf{q}_{\mathbf{2}}\right)\left[\omega\left(\mathbf{q}_{\mathbf{1}}+\mathbf{q}_{\mathbf{2}}, \mu\right)\right] \\
=-4 g_{2} \frac{F_{1}\left(-\mathbf{q}_{\mathbf{2}}, \mathbf{q}_{\mathbf{2}}\right)}{\left(\sqrt{\left(8 \pi^{3}\right.}\right)^{3} \sqrt{2 \omega\left(\mathbf{q}_{\mathbf{1}}, m\right)} \sqrt{2 \omega\left(\mathbf{q}_{\mathbf{1}}+\mathbf{q}_{\mathbf{2}}, \mu\right)} \sqrt{2 \omega\left(-\mathbf{q}_{\mathbf{2}}, m\right)}} .
\end{array}
$$

Substituting the expression (10) into equation (8), the latter, in the rest frame (i.e. the total momentum $\mathbf{Q}=0$ ), simplifies to the single relativistic momentum-space equation

$$
f(\mathbf{q})[2 \omega(\mathbf{q}, m)-E]=4 \pi \alpha m^{2} \int d^{3} \mathbf{p} \frac{f(\mathbf{p})}{\omega(\mathbf{q}, m) \omega^{2}(\mathbf{p}-\mathbf{q}, \mu) \omega(\mathbf{p}, m)} .
$$


where $f(\mathbf{q})=F_{1}(-\mathbf{q}, \mathbf{q})$, and $\alpha=\frac{2 g_{2}^{2}}{(4 \pi)^{4} m^{2}}$ is a dimensionless coupling constant. The interaction in the integral equation (11) is represented by the kernel, which is a relativistic generalization of the potential.

Our principal interest is to determine the conditions under which the twoHiggs system can form bound states due to a Dark Matter mediating field. For this purpose it is sufficient to study ground states, for which the wave functions are spherically symmetric. We shall examine the $(\alpha, \mu)$ parameter space, where $\mu$ is the (unknown) DM particle mass, and $\alpha$ (or, equivalently, $g_{2}$ ), is the (similarly unknown) dimensionless coupling constant.

\section{Approximate solutions and results}

Unfortunately the relativistic equation (11) is not analytically solvable even for spherically symmetric states, so approximate variational solutions will be obtained. Variational approximations, as is well known, are only as good as the trial states that are used. For our purposes it will be sufficient to use simple ground state trial functions, particularly in light of the large parameter space to be examined.

We shall obtain approximate variational solutions of (11) for the HiggsHiggs ground state, using the spherically symmetric trial wave function

$$
f(p)=\frac{\omega(p, m)}{\left(p^{2}+b^{2}\right)^{2}},
$$

where $p=|\mathbf{p}|, \quad \omega(p, m)=\sqrt{\left(p^{2}+m^{2}\right.}$ and $b$ is an adjustable parameter, whose value is chosen so that the $E_{\text {trial }}(b ; \alpha, m, \mu)$ is a least upper bound to the unknown exact mass of the two-Higgs bound state.

It should be noted that for non-relativistic Higgs particles, i.e. in the limit $\frac{p^{2}}{m^{2}} \ll 1$, (equivalently, $\omega(p, m) \rightarrow m$ ), equation (11) simplifies to

$$
f(\mathbf{q})\left[\frac{\mathbf{q}^{2}}{m}-\epsilon\right]=4 \pi \alpha \int \mathrm{d}^{3} \mathbf{p} \frac{f(\mathbf{p})}{\left.(\mathbf{p}-\mathbf{q})^{2}+\mu^{2}\right)},
$$

where $\epsilon=E-2 m$. This is recognized to be the momentum space representation of the non-relativistic Schrödinger equation for the relative motion of two Higgs particles interacting via an attractive Yukawa potential $-\alpha \frac{e^{-\mu r}}{r}$. 
As is well known, this non-relativistic equation is not analytically solvable for $\mu>0$ though in the limit of massless Dark Matter particles, $\mu=0$, the solutions are the familiar Hydrogenic ones with $\epsilon=-\frac{1}{4} m \alpha^{2}$ and $b=\frac{1}{2} m \alpha$ for the ground state.

The variationally obtained relativistic two-Higgs ground-state rest masses $E$ are listed in Table 1 for various values of the dimensionless coupling constant $\alpha$ and for various values of the DM mass $\mu$. All results are given in units of the Higgs mass $m$, i.e. $E$ is $E / m, \mu$ is $\mu / m, b$ is $b / m$.

We also list the previously obtained non-relativistic limit results for comparison purposes [15]. These relativistic and non relativistic results are also plotted in Figure 1 and in Figure 2.

Table 1. Variational relativistic two Higgs rest energy $E_{r e l}$ for various values of the DM mass $\mu$ (both in units of the Higgs mass $m$ ) and for various values of the dimensionless coupling constant $\alpha$. The quantity $b$ is the optimal value of the variational scale parameter $b$ (in units of the Higgs mass $m$ ). We also give the non-relativistic result $E_{n r}$ for comparison purposes.

\begin{tabular}{|l|l|l|l|l|}
\hline \multicolumn{5}{|c|}{$\alpha=0.01$} \\
\hline$\mu$ & $b$ & $b$ & $\begin{array}{l}E \\
\text { Non-Relativistic }\end{array}$ & $\begin{array}{l}E \\
\text { Relativistic }\end{array}$ \\
\hline 0.0000 & 0.0050000000 & 0.0049989444 & 1.999975000 & 1.999975000 \\
0.0010 & 0.0048783984 & 0.0048774111 & 1.999983664 & 1.999983666 \\
0.0015 & 0.0047460883 & 0.0047451485 & 1.999987133 & 1.999987136 \\
0.0020 & 0.0045751120 & 0.0045742278 & 1.999990122 & 1.999990123 \\
0.0025 & 0.0043674490 & 0.0043666272 & 1.999992675 & 1.999992676 \\
0.0030 & 0.0041221774 & 0.0041214241 & 1.999994832 & 1.999994833 \\
0.0035 & 0.0038348722 & 0.0038341925 & 1.999996625 & 1.999996626 \\
0.0040 & 0.0034951351 & 0.0034945326 & 1.999998076 & 1.999998077 \\
0.0045 & 0.0030781877 & 0.0030776632 & 1.999999201 & 1.999999202 \\
0.0050 & 0.0025000000 & 0.0024995329 & 2.000000000 & unbound \\
\hline
\end{tabular}




\begin{tabular}{|c|c|c|c|c|}
\hline \multicolumn{5}{|c|}{$\alpha=0.1$} \\
\hline$\mu$ & $b$ & $\begin{array}{l}b \\
\text { Relativistic }\end{array}$ & $\begin{array}{l}E \\
\text { Non-Relativistic }\end{array}$ & $\begin{array}{l}E \\
\text { Relativistic }\end{array}$ \\
\hline 0.000 & 0.0500000000 & 0.0490491428 & 1.997500000 & 1.997527459 \\
\hline 0.010 & 0.0487839844 & 0.0478935044 & 1.998366353 & 1.998389559 \\
\hline 0.015 & 0.0474608825 & 0.0466114592 & 1.998713376 & 1.998733632 \\
\hline 0.020 & 0.0457511204 & 0.0449494329 & 1.999012120 & 1.999029227 \\
\hline 0.025 & 0.0436744901 & 0.0429265681 & 1.999267453 & 1.999281386 \\
\hline 0.030 & 0.0412217741 & 0.0405329877 & 1.999483229 & 1.999494095 \\
\hline 0.035 & 0.0383487224 & 0.0377237277 & 1.999662509 & 1.999670520 \\
\hline 0.040 & 0.0349513506 & 0.0343937611 & 1.999807643 & 1.999813089 \\
\hline 0.045 & 0.0307818766 & 0.0302924647 & 1.999920155 & 1.999923382 \\
\hline & & $\alpha=0$. & & \\
\hline$\mu$ & $\begin{array}{l}b \\
\text { Non-Relativist }\end{array}$ & $\begin{array}{l}b \\
\text { Relativistic }\end{array}$ & $\begin{array}{l}E \\
\text { Non-Relativistic }\end{array}$ & $\begin{array}{l}E \\
\text { Relativistic }\end{array}$ \\
\hline 0.00 & 0.2500000000 & 0.1954804199 & 1.937500000 & 1.947011993 \\
\hline 0.01 & 0.2497147851 & 0.1957478826 & 1.942353821 & 1.951649179 \\
\hline 0.05 & 0.2439199223 & 0.1923172164 & 1.959158829 & 1.967135921 \\
\hline 0.1 & 0.2287556021 & 0.1809286299 & 1.975303007 & 1.981177410 \\
\hline 0.15 & 0.2061088708 & 0.1631240265 & 1.987080715 & 1.990833047 \\
\hline 0.20 & 0.1747567533 & 0.1375741000 & 1.995191084 & 1.997083715 \\
\hline 0.21 & 0.1669975210 & 0.1310202512 & 1.996412850 & 1.997980449 \\
\hline 0.23 & 0.1490363127 & 0.1151845935 & 1.998469730 & 1.999440329 \\
\hline 0.24 & 0.1381835823 & & 1.999303047 & unbound \\
\hline 0.245 & 0.1319854886 & & 1.999669182 & unbound \\
\hline & & $\alpha=1$. & 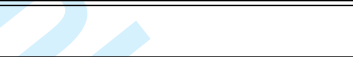 & \\
\hline$\mu$ & $b$ & $b$ & $E$ & $E$ \\
\hline & Non-Relativist & Relativistic & Non-Relativistic & Relativistic \\
\hline 0.0 & 0.5000000000 & 0.3021534764 & 1.750000000 & 1.828329614 \\
\hline 0.01 & 0.4998538419 & 0.3030882883 & 1.759851954 & 1.837268511 \\
\hline 0.1 & 0.4878398446 & 0.2997899929 & 1.836635318 & 1.901360074 \\
\hline 0.3 & 0.4122177417 & 0.2492925688 & 1.948322860 & 1.977746849 \\
\hline 0.4 & 0.3495135065 & 0.2026640762 & 1.980764335 & 1.995117073 \\
\hline 0.42 & 0.3339950421 & 0.1899549310 & 1.985651399 & 1.997375352 \\
\hline 0.45 & 0.3078187666 & & 1.992015541 & unbound \\
\hline
\end{tabular}




\begin{tabular}{|l|l|l|l|l|}
\hline \multicolumn{5}{|c|}{$\alpha=1.5$} \\
\hline$\mu$ & $b$ & $b$ & $E$ & $E$ \\
& Non-Relativistic & Relativistic & Non-Relativistic & Relativistic \\
\hline 0.0 & 0.7500000000 & 0.3728822765 & 1.437500000 & 1.676269261 \\
0.01 & 0.7499017302 & 0.3742905228 & 1.452351313 & 0.372882277 \\
0.1 & 0.7413970995 & 0.3768978968 & 1.573658016 & 1.785482227 \\
0.3 & 0.6862668064 & 0.3462987386 & 1.777727066 & 1.916214889 \\
0.5 & 0.5903671916 & 0.2821499316 & 1.911494893 & 1.980371260 \\
0.6 & 0.5242702599 & 0.2315506632 & 1.956719753 & 1.996503625 \\
0.7 & 0.4368091778 & & 1.988859776 & unbound \\
\hline \hline \multicolumn{5}{|c|}{$\alpha=2.0$} \\
\hline$\mu$ & $b$ & $b$ & $E$ & $E$ \\
\hline 0.00 & 1.000000000 & 0.4255384887 & 1.000000000 & 1.503833978 \\
0.01 & 0.9999259798 & 0.4273308572 & 1.019850988 & 1.520463609 \\
0.10 & 0.9933301511 & 0.4341423237 & 1.185897111 & 1.647178628 \\
0.30 & 0.9492176505 & 0.415127049 & 1.485350682 & 1.830237683 \\
0.50 & 0.8734898018 & 0.3685721652 & 1.706981212 & 1.932649768 \\
0.75 & 0.7345173498 & 0.2697740392 & 1.895690531 & 1.994104525 \\
0.80 & 0.6990270133 & 0.2369773494 & 1.923057339 & 1.999960782 \\
0.90 & 0.6156375332 & \multicolumn{5}{|l|}{} \\
\hline
\end{tabular}




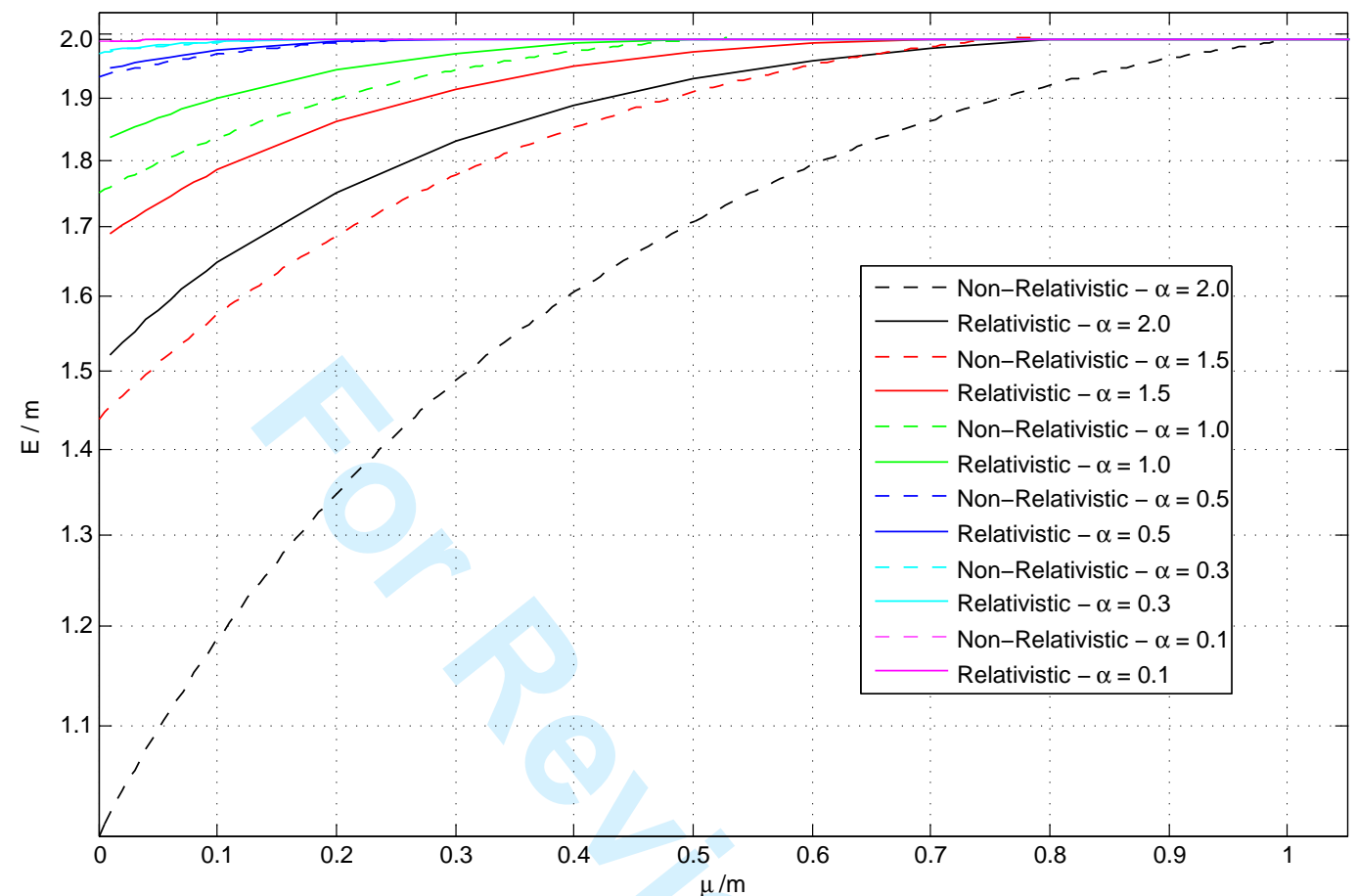

Figure 1: The two-Higgs rest mass $E_{\text {min }}$ versus DM mass $\mu$, in units of the Higgs mass $m$. Relativistic results are represented by the solid curves, dashed curves represent the corresponding non-relativistic calculations.

It is evident that the relativistic values of the bound two-Higgs rest mass $E$ are significantly higher (i.e. the binding energy is significantly lower) than the non-relativistic values, and the difference grows with increasing values of the coupling constant $\alpha$. This underscores the importance of using a relativistic description.

\section{Concluding remarks}

Our results show that two-Higgs bound states can be formed due to a massive spinless real scalar Dark Matter mediating field, over a broad range of the effective dimensionless coupling constant $\alpha=2 g_{2}^{2} /(4 \pi)^{4} m^{2}$ and DM mass $\mu$, namely $0<\alpha \leq 2$ and $\mu \leq m$, i.e. decay of the DM particle into two Higgs particles is not possible. 


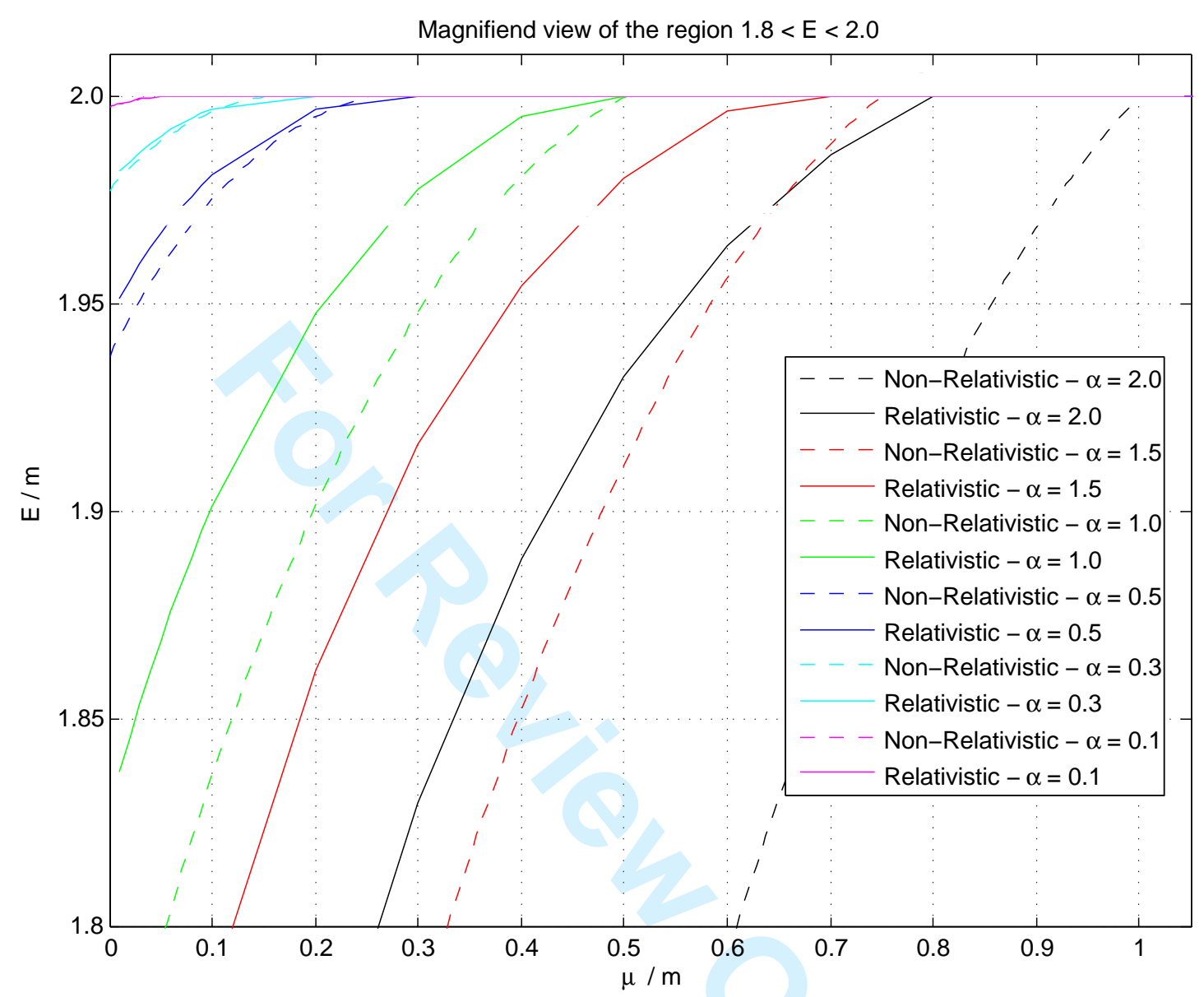

Figure 2: Two-Higgs rest mass $E_{\text {min }}$ versus DM mass $\mu$, in units of the Higgs mass $m$, magnified Figure 1 for the region $1.8<E<2.0$

Note that the indicated domain of the effective dimentionless coupling constant $\alpha$ does not require that $g_{2}$ be large since $\alpha$ is proportional to $\left(g_{2} / m\right)^{2}$.

Of course, these bound states are actually quasi-bound states since the Higgs particle has a very short lifetime and so the two-Higgs bound system is also short lived. Such quasi-bound states are expected to manifest themselves as resonances in the scattering cross section in DM on DM collisions. This is analogous to bound states of positronium, which are also short-lived quasi-bound states of an electron-positron system that manifest themselves as resonances in photon-photon scattering [20]. 
In the present work we do not study $H$ on $H$ elastic scattering states, that is solutions of equation (11) with $E>2 \mathrm{~m}$. Also, the effect of the coupling constants other than $g_{2}$ are not sampled in this work. Their effect on the binding energies can be evaluated perturbatively or by expanding the number of Fock components in the trial state $\left|\psi_{\text {trial }}\right\rangle$. In any case, their effect would not eliminate the binding, since the interactions in this model are overall attractive. We shall report on the effect of interaction terms of the Lagrangian (1) with coupling constants other than $g_{2}$ in subsequent work.

\section{References}

[1] F. Zwicky (1933) Die Rotverschiebung von extragalaktischen Nebeln Helvetica Physica Acta 6, 110; and (1937) On the Masses of Nebulae and of Clusters of Nebulae, The Astrophysical Journal 86217.

[2] V. Trimble (1987) Existence and nature of dark matter in the universe, Annual Review of Astronomy and Astrophysics 25, 425.

[3] G. Bertone, D. Hooper and J. Silk (2005) Particle dark matter: evidence, candidates and constraints, Phys. Reports 405, 279

[4] L. Baudis (2013) A review of direct WIMP search experiments, Nuclear Physics B - Proceedings Supplements 235-6, 405.

[5] R. F. Lang (2013) Viewpoint: Ups and Downs in the Search for Dark Matter, Physics 6, 136.

[6] T. Hambye and M. H. G. Tytgat (2010) Confined hidden vector dark matter, Phys. Letters B 683, 39.

[7] A. Crivellin, U. Haisch and A. Hibbs (2015) LHC constraints on gauge boson couplings to dark matter, Phys. Rev. D 91, 074028.

[8] A. Berlin, D. Hooper and S. D. McDermott (2015) Dark matter elastic scattering through Higgs loops, Phys. Rev. D 92, 123531.

[9] C. Petersson, A. Romagnoni and R. Torre (2012) Higgs Decay with Monophoton + MET Signature from Low Scale Supersymmetry Breaking, J. of High Energy Physics, 10, 016. 
[10] R. N. Cahn and M. Suzuki (1984) The Higgs-Higgs bound State, Physics Letters B 134, 115.

[11] J. A. Grifols (1991) Higgsonium, Physics Letters B 264, 149.

[12] L. Di Leo and J.W. Darewych (1994) Bound States in the Higgs Model, Phys. Rev. D, 49, 1659.

[13] F. Siringo (2000) Variational study of bound states in the Higgs model, Phys. Rev. D 62, 116009.

[14] G. Grinstein and M. Trott (2007) Higg-Higgs bound state due to new physics at a TeV, Phys. Rev. D B 76, 073002.

[15] Y. Bhattacharya and J. W. Darewych (2016) Higgs particles interacting via a scalar Dark Matter field, EPJ Web of Conferences, 113, 08021.

[16] J. M. Cline, P. Scott, K. Kainulainen and C. Weniger (2013) Update on scalar singlet dark matter, Phys. Rev. D 88, 055025.

[17] A. Bernal, J. Barranco, D. Alic and C. Palenzuela (2010), Galactic dark matter halo made of spin zero bosons, AIP Conf. Proc. 1241, 335.

[18] S. Esch, M. Klasen and C. E. Yaguna (2014) A minimal model of twocomponent dark matter, J. of High Energy Physics, 2014,108.

[19] T. Hambye, F.-S. Ling, L. Lopez-Honorez and J. Rocher (2009) Scalar multiplet dark matter, J. High Energy Physics, 2009, 07, 090.

[20] J. W. Darewych, M. Horbatsch and R. Koniuk (1992) Photon-Photon Resonances in Quantum Electrodynamics, Phys. Rev. D 45, 675.

[21] O. Cata and A. Ibarra (2014) Dark matter stability without new symmetries, Phys. Rev. D 90, 063509.

[22] W. Rodejohann and C. E. Yaguna (2015) Scalar dark matter in the B - L model, J. of Cosmology and Astroparticle Physics 12, 032.

[23] M. Cirelli, (2012) Dark Matter searches: A theoretical perspective, Nuovo Cim. 35 C, N. 6, 063509. 